\title{
Médiévales
}

Langues, Textes, Histoire

70 | printemps 2016

Lieux d'hygiène et lieux d'aisance en terre d'Islam (VII $\mathrm{e}_{-}$ $\mathrm{XV}^{\mathrm{e}}$ siècle)

\section{Lieux d'aisance et de toilette en milieu rural dans le Maroc médiéval. L'exemple des latrines du site d'Igīìz et les déchets des plantes consommées}

Toilet and Bathroom in Rural Areas of Medieval Morocco: the Example of the Latrines of the Site of Igiliz and the Food Plant Waste

Jean-Pierre Van Staëvel, Marie-Pierre Ruas, Ahmed S. Ettahiri et Abdallah Fili

\section{OpenEdition} Journals

Édition électronique

URL : http://journals.openedition.org/medievales/7848

DOI : $10.4000 /$ medievales.7848

ISSN : $1777-5892$

Éditeur

Presses universitaires de Vincennes

Édition imprimée

Date de publication : 23 juin 2016

Pagination : 189-213

ISSN : 0751-2708

Référence électronique

Jean-Pierre Van Staëvel, Marie-Pierre Ruas, Ahmed S. Ettahiri et Abdallah Fili, « Lieux d'aisance et de toilette en milieu rural dans le Maroc médiéval. L'exemple des latrines du site d'Igilïz et les déchets des plantes consommées », Médiévales [En ligne], 70 I printemps 2016, mis en ligne le 15 juin 2018, consulté le 20 avril 2019. URL : http://journals.openedition.org/medievales/7848 ; DOI : 10.4000/ medievales.7848 
Jean-Pierre Van Staëvel, Marie-Pierre Ruas, Ahmed S. Ettahiri et Abdallah Fili

\section{Lieux d'aisance et de toilette en milieu rural dans le Maroc médiéval \\ L'exemple des latrines du site d'lgīlīz et les déchets des plantes consommées}

Épicentre initial de la révolution almohade au début des années 1120 , le site d'Igīlīi ${ }^{1}$ reste attaché à la mémoire du juriste-théologien et fondateur du mouvement Ibn Tūmart ${ }^{2}$. Depuis sa découverte en 2004, il nous a été donné à plusieurs reprises de revenir sur le rôle exceptionnel qu'a joué la montagne d'Igīlīz dans l'histoire du Maroc médiéval et du Maghreb tout entier ${ }^{3}$. Rapidement marginalisé du temps de l'Empire almohade, puis ramené aux dimensions d'un modeste village avant d'être finalement déserté, ce site offre donc à l'archéologue la chance unique de pouvoir étudier de larges pans de la culture matérielle d'une communauté montagnarde dans une contrée périphérique et encore largement méconnue par les archéologues et les historiens des textes. Mené dans le cadre d'une action de coopération scientifique entre la France et le Maroc, le programme La Montagne d'Igìlīz et le pays des Arghen, enquête archéologique sur les débuts de l'empire almohade compte à ce jour six campagnes de fouille ${ }^{4}$. Les matériaux déjà

1. Pour des raisons liées à l'usage linguistique local, les translittérations des termes arabes et amazighs (tachelhit) sont parfois simplifiées. Les toponymes suivent l'orthographe des cartes, ou la transcription d'usage local.

2. Sur l'historiographie du site, nous nous permettons de renvoyer à : J.-P. VAN STAËVEL, A. FiLI, «Wa-wașalnā 'alā barakat Allāh ilā İgīlīz: à propos de la localisation d'Igīlīz-des-Harg̉a, le hiṣn du Mahdī Ibn Tūmart », Al-Qanțara, 17 (2006), p. 153-194.

3. On en trouvera une récente présentation d'ensemble dans A. S. ETTAHIRI, A. FILI, J.-P. VAN STAËVEL, « Nouvelles recherches archéologiques sur les origines de l'Empire almohade au Maroc : les fouilles d'Igīlīz », Comptes rendus des séances de l'Académie des Inscriptions et Belles-Lettres 2013, 2 (2014), p. 1053-1086.

4. La mission archéologique Igīlīz a été créée en 2009 par le ministère des Affaires Étrangères, avec le soutien décisif de la Casa de Velázquez. Ce programme est dirigé par A. Fili, A. S. Ettahiri et J.-P. Van Staëvel. Il bénéficie du support institutionnel et du soutien financier de divers partenaires : le ministère des Affaires étrangères et européennes, la Casa de Velázquez, l'Institut National des Sciences de l'Archéologie et du Patrimoine à Rabat, les UMR 8167 (Orient et Méditerranée), 5648 (CIHAM) et 7209 (Muséum national d'Histoire 
rassemblés sont éloquents. Ils permettent de dater l'occupation principale du site du XII ${ }^{\mathrm{e}}$ siècle, même si de récentes découvertes permettent dorénavant d'étendre à la fois vers l'amont et vers l'aval une fréquentation du site dont les modalités restent encore à préciser.

Située à $60 \mathrm{~km}$ à l'est sud-est de Taroudant, dans l'Anti-Atlas central, la montagne d'Igīlīz se présente comme un piton presque totalement isolé des massifs qui l'entourent. Protégé par un relief très escarpé, le site archéologique culmine à $1350 \mathrm{~m}$ d'altitude. Il comprend un système défensif composé de murailles et de portes, une grande résidence de chef - la Qașba - ainsi que d'autres vestiges monumentaux - notamment ceux d'une grande-mosquée et d'aménagement cultuels et rituels autour de deux « grottes » (fig. 1).

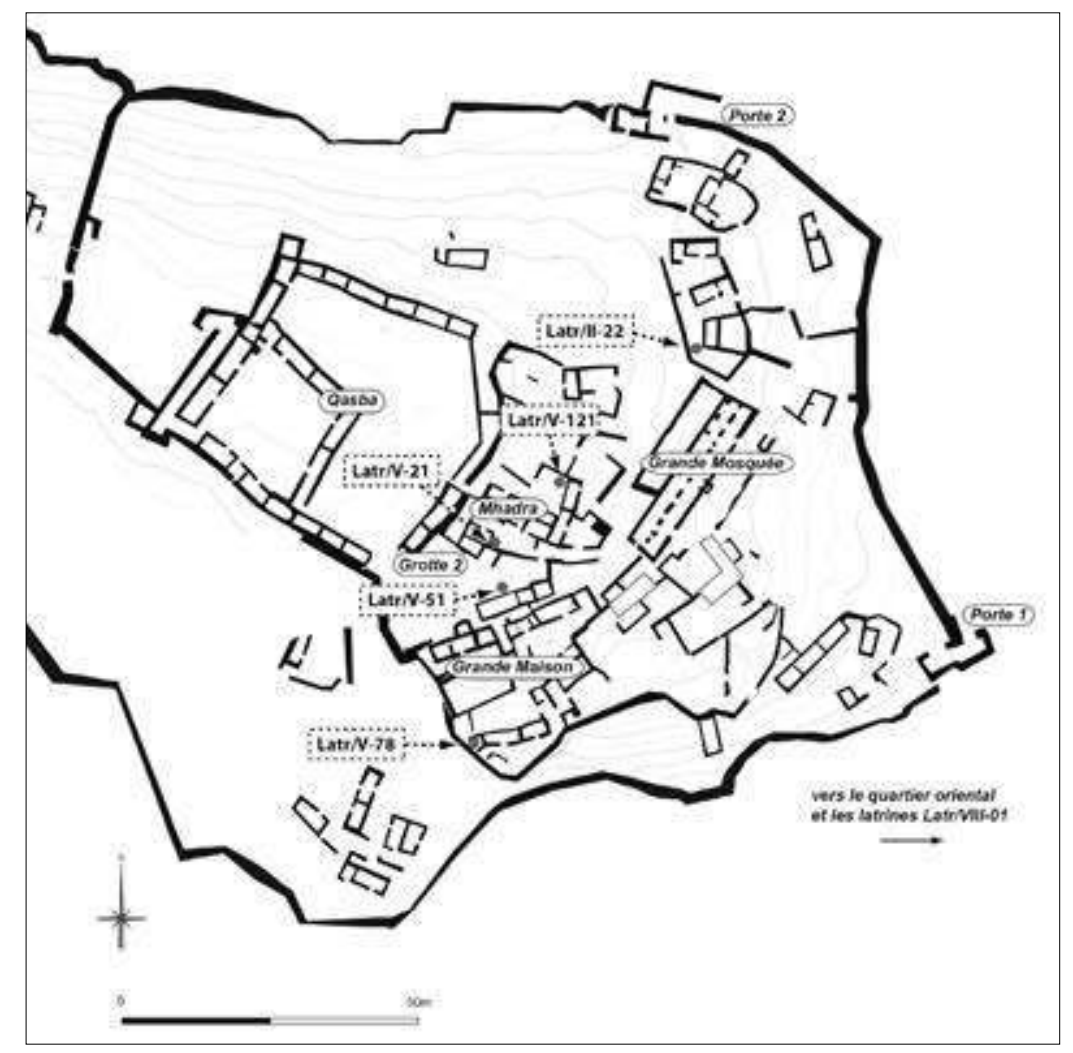

Fig. 1. Plan partiel de l'acropole d'Igīlìz (état 2012)

et emplacement des lieux d'aisance mentionnés dans le texte

(C) R. Schwerdtner, Mission archéologique Igīlīz)

naturelle). Le programme implique également les Universités de Paris-Sorbonne et d'El Jadida, le Labex Resmed et le Centre Jacques Berque à Rabat. 
Le site constitue par ailleurs un poste d'observation d'une importance primordiale pour détailler les structures d'habitat d'une société rurale et tribale, dans une région pour laquelle nos connaissances sur les campagnes médiévales et prémodernes sont quasi inexistantes. L'habitat, dont l'occupation maximale se situe entre la fin $\mathrm{du} \mathrm{XI}^{\mathrm{e}}$ siècle et la fin du siècle suivant (ou peu après), se concentre principalement en trois endroits : au niveau de l'acropole, où plusieurs secteurs de maisons sont en cours de fouille en contrebas du complexe résidentiel de la Qașba, autour de la grande mosquée et des « grottes »; sur les premières pentes méridionales de la montagne en dessous du sommet, là où se déploie le principal quartier d'habitations ; enfin, le long de la crête du sommet le plus oriental, parsemé de constructions dispersées. La fouille s'est, pour le moment, essentiellement cantonnée à l'étude de l'habitat sur l'acropole ; l'exploration des quartiers extra-muros ne fait que commencer. Les pièces de vie se groupent parfois autour de cours communes : c'est notamment le cas de l'ensemble dit de la «Mḥadra », situé en contrebas de la Qașba. Plus généralement toutefois, les maisons monocellulaires s'implantent selon les courbes de niveau, ouvrant chacune sur une cour formant une petite plate-forme en surplomb de l'habitation suivante. Parfois enfin, les unités d'habitation forment de véritables files de trois à quatre pièces qui structurent fortement l'organisation spatiale d'ensemble du site. Si, par bien des indices matériels, ces maisons renvoient à une société agropastorale et à une culture matérielle issue du monde rural, cette dimension de ruralité se doit d'être néanmoins questionnée : sur ce site dont les textes rapportent qu'il a abrité, au début du mouvement almohade, une communauté charismatique assez hétéroclite, la question d'éventuels apports venant de l'extérieur, et reflétant notamment divers aspects de la culture matérielle citadine, ne saurait en effet être négligée. Dans ce contexte, la présence de latrines ou de salles d'eau en différents points de la montagne d'Igīlīz revêt une importance particulière. On s'attachera donc, dans cette contribution qui s'appuie sur des données encore très provisoires, à mieux cerner les contours de ces aménagements destinés à la satisfaction des besoins corporels, à la toilette intime et à l'accomplissement des ablutions obligatoires. On montrera ensuite comment l'approche archéobotanique permet de tirer profit des restes conservés dans ces latrines, éclairant ainsi les pratiques de production et de consommation en usage à Igīlīz durant l'époque médiévale. 


\section{Latrines et systèmes sanitaires sur la montagne d'Igïlīz}

\section{Localisation des latrines sur le site}

À Igīlīz, une première appréciation générale permet de considérer la présence de latrines dans les espaces domestiques comme une exception plutôt que la règle. On laissera tout d'abord de côté la petite salle d'eau (Abl/IV-15) située dans l'aile sud-ouest de la zone de commandement de la Qașba. Cette pièce, dotée d'une banquette latérale et dont le sol et les murs étaient revêtus d'un mortier maigre, possède également un exutoire se déversant, à partir de l'extrémité sud-ouest de la banquette, à l'extérieur de l'enceinte. L'originalité du dispositif semble exclure une utilisation directe en tant que latrines, et il est beaucoup plus probable qu'il s'agissait d'un local à ablutions, depuis lequel pouvaient être éventuellement rejetés à l'extérieur les déchets produits par les habitants du lieu. On écartera également d'emblée de l'analyse les deux salles à ablutions qui ont été retrouvées à proximité de la mosquée de la Qaṣba et du lieu de culte principal. Si l'on s'en tient donc aux latrines stricto sensu, six aménagements de ce type ont été identifiés sur le site à ce jour (fig. 1). La première cour de la Mḥadra abrite des lieux d'aisance (Latr/V-21) dans sa partie sud-ouest : ceux-ci se logent entre le mur de clôture du complexe résidentiel et une pièce de vie (fig. 2 a-b). Abritées par un petit mur qui les isolait de la vue depuis les autres pièces ouvrant sur la cour, ces latrines devaient être couvertes par une toiture légère que supportaient, au nord, deux poteaux dont on a retrouvé les traces d'ancrage au sol. Non loin de là, de l'autre côté du mur de clôture de la Mḥadra, s'étend une esplanade qui commandait l'accès à la grotte $2:$ c'est là qu'ont été installées d'autres latrines (Latr/V-51, fig. 2c), dont le réduit inscrit dans un arc de cercle s'adosse à des maisons ; sans communication avec celles-ci, l'aménagement doit probablement être mis en relation avec la grotte 2 et les visites pieuses auxquelles elle donnait lieu. En contrebas du secteur de la grotte, vers le sud, se déploie un secteur d'habitat dense autour de la "Grande Maison » : c'est là que se trouve, en bordure d'un à-pic, une maison possédant ses propres latrines (Latr/V-78). En contact direct avec le rempart qui le limite au sud, l'espace en question forme un lieu de service exigu de plan trapézoïdal, auquel on accédait au moyen de deux marches. Malgré un état très dégradé, l'aspect général de ces latrines témoigne encore du soin apporté à leur réalisation. Toujours au niveau du sommet de la montagne mais plus au nord-est, au-delà de la grande mosquée et de sa zone de services et en allant vers la porte 2 , se trouve un autre secteur d'habitat fortement marqué par les activités rurales : c'est là que d'autres latrines (Latr/II-22) ont été retrouvées en 2013, dans un petit réduit de plan irrégulier qui n'a été que partiellement fouillé. À ce premier lot de structures identifiées comme des latrines sont venus s'ajouter, au printemps 


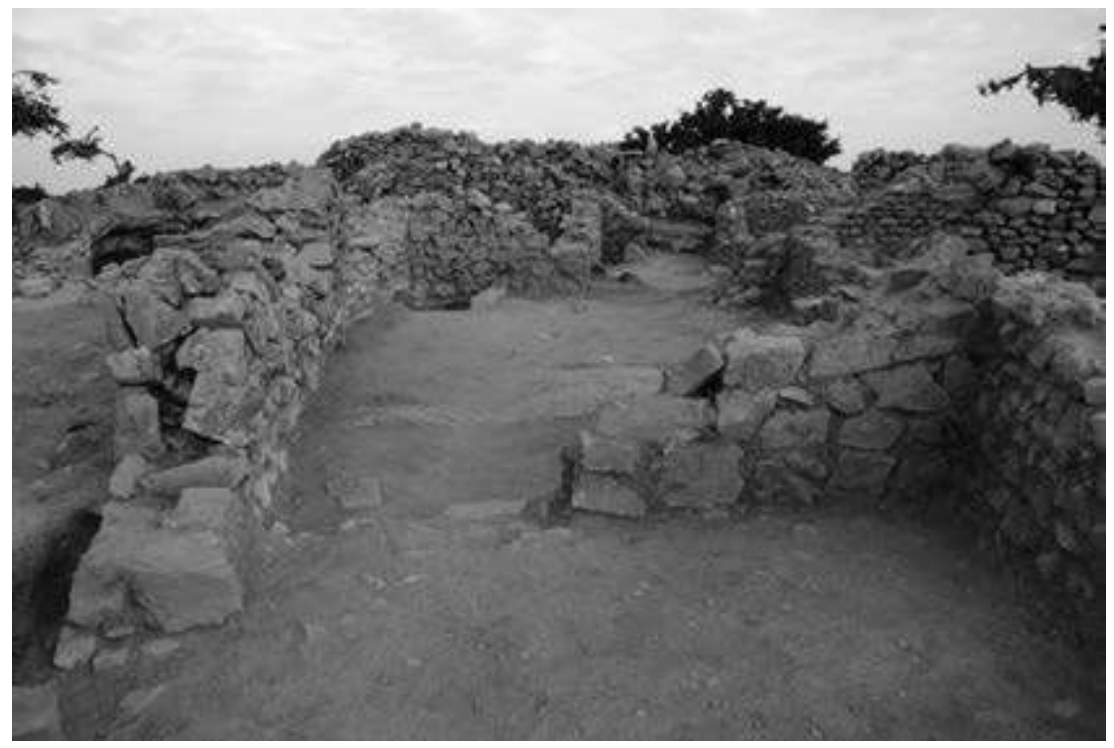

Fig. 2a. Acropole d'Igīlīz, Mhadra : vue d'ensemble de la première cour, avec les latrines au fond à gauche

(C) Mission archéologique Igīlīz)

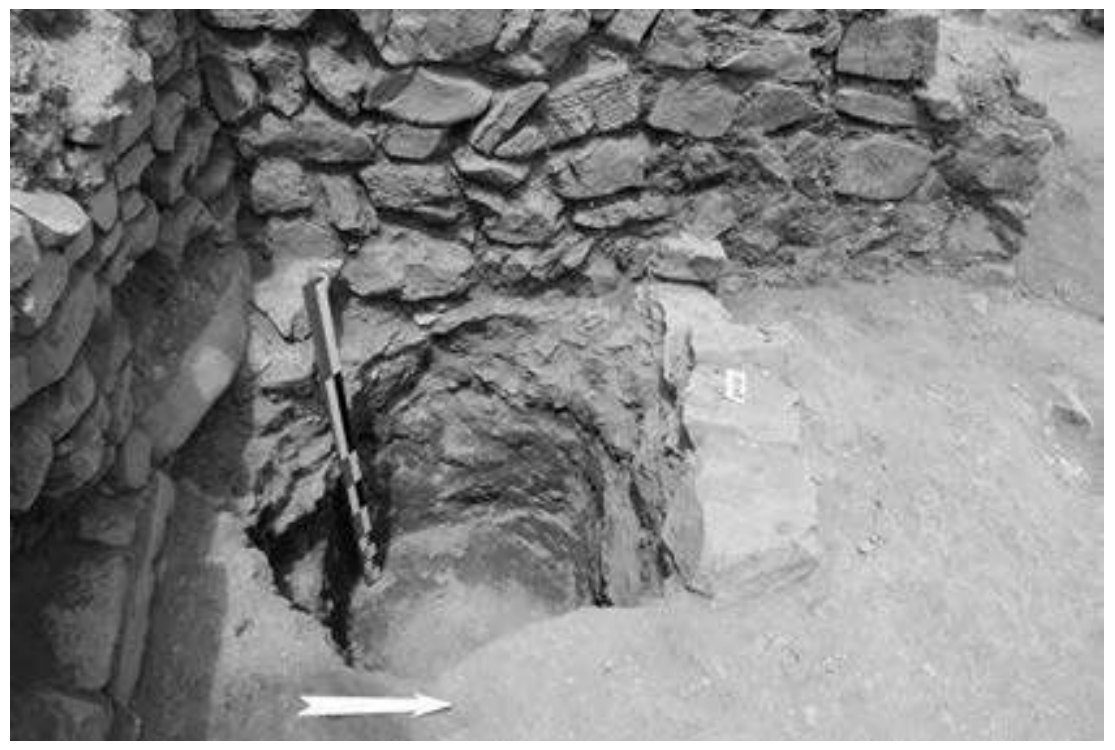

Fig. 2b. Acropole d'Igīlīz, Mḥadra : vue d'ensemble de la fosse des latrines (C) Mission archéologique Igīlīz) 
2014, deux nouveaux spécimens qui ont été retrouvés, respectivement, dans la zone de services en arrière du lieu de culte principal (Latr/V-121), et dans le grand quartier d'habitat extra-muros qui occupe les premières pentes au sud-est de l'acropole (Latr/VIII-01, fig. 2d).

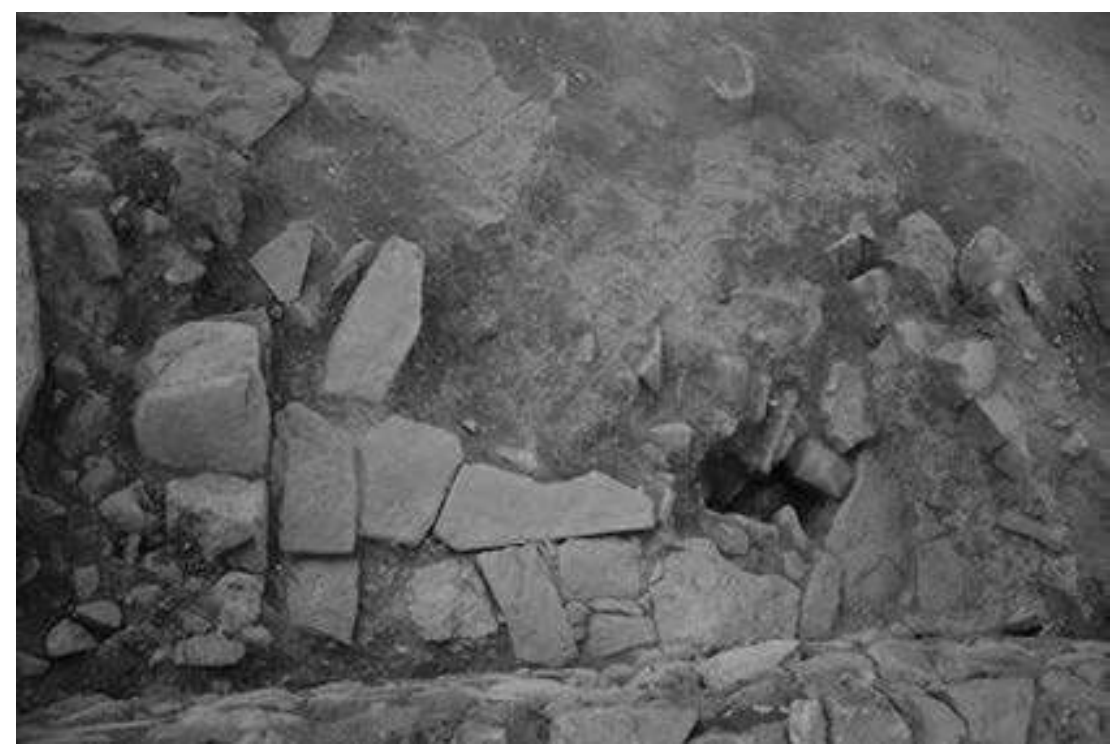

Fig. 2c. Acropole d'Igīlīz, Latr V-51 : vue d'ensemble des latrines

(C) Mission archéologique Igīlīz)

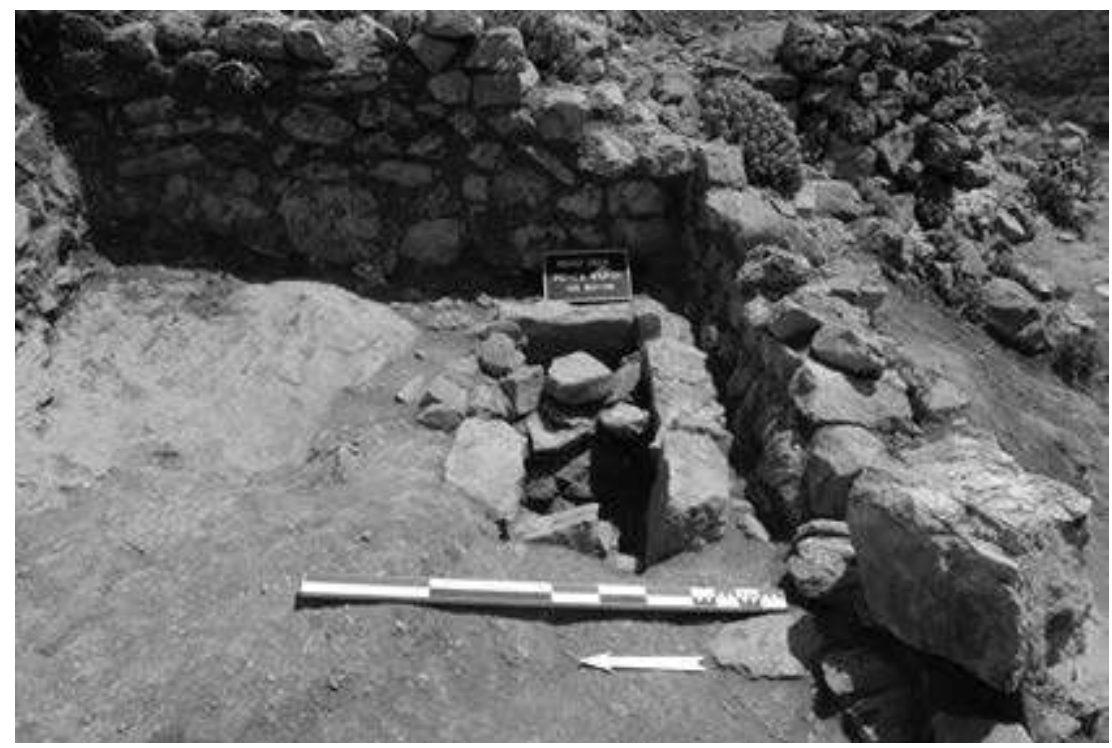

Fig. 2d. Quartier oriental, Latr VIII-01 : vue d'ensemble des latrines

(C) Mission archéologique Igīlīz) 


\section{Des formes diverses}

L'intérêt de ces découvertes sur le site d'Igīlīz consiste notamment dans la diversité morphologique des structures interprétées comme des lieux d'aisance et de toilette intime. Il convient certes de regretter l'état de conservation assez médiocre avec lequel la plupart de ces aménagements nous sont parvenus, et qui nous empêche parfois d'en restituer les détails. Deux d'entre eux ont fait l'objet de pillages, et leurs structures ont été fortement altérées : c'est le cas notamment de la structure Latr/V-51, construite initialement avec grand soin. Les plates-formes initiales - si tant est qu'elles aient été toutes construites en dur, ce qui reste à prouver pour les fosses de la Mhadra (Latr/V-21) et du secteur d'habitat septentrional (Latr/ II-22) - semblent avoir été fortement sollicitées par les récupérateurs de blocs et de dalles à la fin du Moyen Âge ou au début de l'époque moderne, au moment où l'on note une timide tentative de recolonisation de l'espace de l'agglomération médiévale par quelques maisons paysannes.

Nonobstant ce problème de conservation, on constate de grandes différences de formes, de traitement et de soin apportés à la construction de ces aménagements. Trois types de latrines peuvent ainsi être mis en évidence. Seuls deux d'entre eux présentent un aspect rappelant étroitement les équipements connus dans les maisons urbaines du monde islamique occidental : la plate-forme, constituée d'un dallage ou d'un sol de chaux, ménage une fente de plan rectangulaire plus ou moins allongée par où sont évacuées la miction et les fèces. Tel est le cas des latrines situées près de l'àpic sud (Latr/V-78) et de la grotte 2 (Latr/V-51). Les premières présentent une plate-forme intérieure uniformément recouverte d'une couche de chaux ; l'orifice rectangulaire est en communication avec une canalisation qui se prolonge sous le mur d'enceinte pour déverser les eaux usées en contrebas dans la pente. Les secondes comportaient initialement une plateforme composée de dalles de forme irrégulière agencées avec soin. L'orifice était délimité par une série de cailloux disposés verticalement selon le principe des orthostates, définissant un canal rectangulaire de $0,18 \mathrm{~m}$ de largeur pour 0,90 $\mathrm{m}$ de longueur. La base des murs porte encore un enduit préparatoire en terre, revêtu à l'origine d'une couche de mortier maigre de couleur blanchâtre. Dans les deux cas, un léger pendage donné à la plateforme facilitait le drainage des eaux de la toilette vers l'orifice d'évacuation.

Les structures dégagées sur le site en 2014 présentent un aspect très différent. Creusées directement dans le sol non loin d'un angle de la pièce et dans le sens longitudinal de celle-ci, ces latrines adoptent le plan d'un grand rectangle allongé, mesurant environ 1,20 m de longueur, pour une largeur allant de 0,75 m (Latr/VIII-01) à $1 \mathrm{~m}$ (Latr/V-121). Bien mieux conservées que les autres, celles situées dans une pièce du quartier extra-muros montrent encore les traces évidentes d'une subdivision en deux logements 
au moyen de moellons. Si les pierres de format parallélépipédique qui composent ces aménagements témoignent du soin apporté aux choix des matériaux les délimitant, il n'en va pas de même du traitement d'ensemble de ces latrines : on ne note ainsi ni enduit protecteur, ni même de liant de chaux entre les pierres. Une semblable disposition pourrait se retrouver également dans les lieux d'aisance du secteur d'habitat intra-muros septentrional (Latr/II-22), bien qu'il faille attendre la fin de la fouille pour avoir ou non confirmation de son appartenance à ce groupe morphologique. En l'état de nos connaissances, il parait quelque peu difficile de pouvoir mettre en relation ces structures rectangulaires avec d'autres aménagements analogues. On notera toutefois, en guise de premier élément de réflexion, la similitude qu'elles semblent entretenir avec les latrines de la maison II du quartier d'époque almohade de la Alcaçova à Mértola ${ }^{5}$, et avec certains des aménagements visibles dans la salle d'ablutions de la mosquée de Chellah à l'époque mérinide.

La dernière latrine présentée ici n'apparaît guère plus que comme une fosse, creusée dans la cour de la Mḥadra. Cette fosse, dont nul vestige d'arrachement d'une structure rectangulaire ne vient marquer les bords, offre un diamètre de $0,80 \mathrm{~m}$ de moyenne pour $0,60 \mathrm{~m}$ de profondeur. Aucune trace ne subsiste non plus de la plate-forme supérieure.

\section{Fonctionnement: des questions encore irrésolues}

Malgré la diversité morphologique qui les caractérise, les latrines d'Igīlīz ont pour point commun de ne pas être associées à un système d'évacuation des matières. Seul l'espace 78, en bordure de l'à-pic sud, tranche nettement par sa configuration d'ensemble, qui montre que la réalisation du conduit menant de la rigole de collecte à l'exutoire situé de l'autre côté du rempart, avait été planifiée dès l'implantation des bâtiments dans ce secteur. L'ensemble a donc été remarquablement conçu en fonction de l'espace disponible et du microrelief, et témoigne d'un réel savoirfaire. Tout laisse à penser que les autres lieux d'aisance ont été quant à eux mis en place en fonction des bâtiments existants, et dans l'espace laissé disponible. Contrairement à ce que l'on peut observer dans la plupart des latrines de type urbain, où un conduit passant sous (ou dans) le mur de la maison permet l'écoulement des matières depuis les lieux d'aisance jusque dans le puits perdu situé généralement dans la rue, il n'y a donc, pour les cinq autres spécimens exhumés sur le site d'Igīlīz, pas de dissociation

5. Outre le fait qu'elles présentent un plan rectangulaire très semblable, ces latrines montrent également plusieurs dalles de couverture encore en place ; S. MACIAS, Mértola. Le dernier port de la Méditerranée, Mértola, 2006 (3 vol.), t. 3, p. 69, fig. VI.21 (plan), et p. 85 fig. VI.46 (photo). 
topographique entre la plate-forme et la fosse de collecte ; celle-ci est tout simplement positionnée directement sous la première, dans l'emprise même de l'espace des latrines ${ }^{6}$. Dans ce cas de figure, la vidange devait s'effectuer par la source même des émissions. Les fosses atteignent des dimensions respectables dans deux des cas évoqués (Latr/V-21 et Latr/II-22, celle-ci montrant des signes évidents d'au moins un curage); par contre, elles sont peu développées dans le cas des structures rectangulaires Latr/V-121 et Latr/VIII-01. Les parties supérieures manquantes au-dessus de la fosse de la Mḥadra étaient peut-être en bois ; cette hypothèse a en tout cas été proposée pour pallier les lacunes constatées sur le site espagnol de Villa Vieja de Calasparra ${ }^{7}$. Dans les latrines de structure rectangulaire qui ont été mises au jour dans le secteur d'habitat extra-muros, la partition encore visible en deux logements laisse penser à une division fonctionnelle entre le lieu d'aisance à proprement parler et l'espace où l'on accomplit sa toilette et, vraisemblablement, ses ablutions. La structure ne présente pas de système d'évacuation : là encore, le curage devait s'effectuer depuis la plate-forme. On notera par ailleurs l'existence d'un foyer dans cette pièce : celui-ci ne semble pas avoir eu de fonction culinaire, mais devait plutôt servir à faire chauffer l'eau destinée à la toilette. La structure retrouvée dans la zone de service de la mosquée principale devait vraisemblablement obéir au même principe de fonctionnement.

L'ensemble de ces installations mises au jour à Igīlīz témoigne donc d'expériences techniques diverses, peut-être parfois mal maîtrisées. Un réel savoir-faire en la matière, sans doute importé d'un milieu citadin où la réalisation de telles installations était monnaie courante, n'apparaît vraiment que dans le cas des latrines situées au bord de l'à-pic, tout au sud de l'acropole. Force est également de reconnaître que nous ignorons dans une large mesure comment étaient utilisées ces latrines : les comparaisons archéologiques ne sont généralement possibles que sur le plan de la typologie formelle et du système d'évacuation et/ou de curage, et les ethnologues se sont bien gardés de décrire ces gestes intimes auxquels eux-mêmes pourtant ne pouvaient échapper : cette pudeur excessive nous prive malheureusement d'éléments de compréhension pourtant indispensables.

6. À Cieza, les fosses situées à l'intérieur des maisons - cas rare - sont toutes attribuées à une mise en place en limite de l'espace domestique, avant que celui-ci n'étende dans un second temps son emprise au-dessus du puits perdu en question ; J. NAVARRO PALAZÓN et P. JimÉneZ CASTILlo, Siyâsa. Estudio arqueologico del despoblado andalusi (siglos IX-XIII), Murcie, 2007, p. 192. À Mértola, on rencontre également quelques fosses dans les maisons ; celles-ci sont mises en relation avec des phases d'occupation antérieures ; S. MACIAS, Mértola. Le dernier port de la Méditerranée..., t. I, p. 378.

7. I. POZO MARTíneZ, « La alquería islámica de Villa Vieja (Calasparra, Murcia) », dansA. BAZZAnA et É. Hubert éd., Castrum 6. Maisons et espaces domestiques dans le monde méditerranéen au Moyen Âge, Rome/Madrid, 2000, p. 165-175 (p. 175). 


\title{
Échos de l'alimentation végétale des utilisateurs des latrines
}

\author{
Intérêts et limites de l'étude du contenu organique des latrines
}

Intégrées dans le programme dès 2009, les recherches archéobotaniques (semences et bois) ont apporté des éclairages inédits sur les ressources végétales exploitées, l'alimentation, les pratiques agraires, l'approvisionnement en bois, le terroir de la population médiévale et la reconnaissance d'activités au sein des différents espaces dégagé ${ }^{8}$. En raison des conditions aérobies des niveaux archéologiques, les prélèvements de sédiments ont privilégié les contextes à rejets carbonisés (foyers, fours, niveaux incendiés, vidanges cendreuses, etc.). Mais dans certains contextes moins cendreux, notamment les latrines et d'autres dépotoirs, les vestiges organiques étaient aussi conservés dans un état minéralisé.

Parmi les dix secteurs étudiés (120 unités stratigraphiques), le contenu de trois des six latrines décrites a été prélevé et analysé : Latr/V-21 dans la première cour de la Mḥadra, Latr/V-51 près de la grotte 2 et Latr/ VIII-01 dans une maison du secteur d'habitat extra-muros. Grâce à la mise en évidence de restes de plantes dans le contenu résiduel de ces fosses, leur utilisation et les traits alimentaires des utilisateurs peuvent être appréhendés.

En effet, ce type de réceptacles est connu pour recevoir les excréments, les reliefs de table, les litières, paillasses ou fumiers, les détritus des sols d'habitats et les cendres de foyers culinaires. Ils sont ainsi des sources d'information directes pour la connaissance de l'alimentation et d'autres pratiques liées à l'assainissement des lieux de vie. Ces rebuts domestiques et le piégeage naturel des poussières emprisonnent une grande diversité d'éléments végétaux et animaux ${ }^{9}$. Au sein des rejets végétaux, les semences de nombreuses plantes cultivées et sauvages sont les macro-restes les plus fréquents sous la forme de graines, de pépins, de noyaux ou de fragments de coque. De la paille et du bois, voire des fragments de feuilles, de tiges ou de racines peuvent y être associés. Des pollens et des spores sont aussi présents. Dans les conditions physico-chimiques de la plupart des couches archéologiques en milieu aérobie, la majorité des éléments végétaux accumulés à l'état « frais » sont détruits par divers organismes (champignons, bactéries, insectes, etc.) qui se nourrissent des matières en décomposition. Des événements et des conditions spécifiques de fossilisation peuvent néanmoins assurer leur maintien dans le sédiment : carbonisation

8. M.-P. Ruas, M. Tengberg, A. S. Ettahiri, A. Fili, J.-P. Van StaëVel, «Archaeobotanical Research at the Medieval Fortified Site of Îgîlîz (Anti-Atlas, Morocco) with Particular Reference to the Exploitation of the Argan Tree », Vegetation History Archaeobotany, 20 (2011), p. 419-433.

9. J. GreIG, « Garderobes, Sewers, Cesspits and Latrines », Current Archaeology, 85 (1982), p. 45-52. 
(grillage, incendie), immersion (ou imbibition), minéralisation, dessiccation (ou momification). Dans les latrines, lorsque la partie organique accumulée est constamment immergée par les eaux déversées et la nappe phréatique, les conditions anaérobies favorisent leur conservation dans un état proche de leur forme de rejet. Le déversement de vidanges cendreuses y apporte aussi des semences et des bois carbonisés. Si un assèchement du remplissage survient, les éléments organiques rejetés à l'état frais disparaissent ou sont, pour une part, minéralisés par la précipitation de sels minéraux comme le phosphate de calcium ${ }^{10}$. Bien que la détermination de semences minéralisées soit rendue difficile, elles sont parfois les seuls témoins alimentaires qui persistent dans le réceptacle. La dégradation différentielle des éléments organiques pendant la minéralisation modifie la composition végétale des ensembles rejetés à l'origine, à la fois en type de restes et en espèces ${ }^{11}$. Aussi les quantités de semences et les plantes enregistrées dans ce qu'il reste des remplissages minéralisés ne reflètent pas la composition des repas ni les proportions entre les denrées consommées. Bien que biaisée, une image des ingrédients ingérés demeure appréhendable et complète celle délivrée par les dépôts carbonisés dont les contenus sont les résidus d'activités de transformations réalisées en amont des repas et utilisés in fine comme combustibles. Les semences et autres parties de plantes rejetées dans ces réceptacles sans avoir été carbonisées ont donc de fortes probabilités d'avoir été ingérées pour la plupart; elles témoignent directement des plantes de l'alimentation quotidienne ou particulière.

\section{Les informations archéobotaniques des latrines d'lgīlīz}

Les semences et les charbons de bois ont été extraits par tamisage sous eau des sédiments au travers de tamis à maille fine entre $2 \mathrm{~mm}$ et $0,25 \mathrm{~mm}^{12}$. Le site a livré les vestiges de près de 102 plantes parmi lesquelles 26 espèces étaient cultivées ou sauvages mais exploitées pour leur bois ou leurs fruits d'intérêt alimentaire, technique ou médicinal, et 76 autres plantes sauvages

10. F. J. GREEN, «Phosphatic Mineralization of Seeds from Archaeological Sites », Journal of Archaeological Sciences, 6 (1979), p. 279-284 ; S. PREISS, Exploitation des ressources végétales et alimentation dans le Nord de la France entre les $X^{e}$ et XIVe siècles : études carpologiques de la motte castrale de Boves (Amiens, Somme) et des sites environnants, Doctorat de l'Université de Picardie Jules Verne, 2011.

11. K.-H. KNÖRZER, « Aussagemöglichkeiten von paläoethnobotanischen Latrinenuntersuchungen », dans W. VAN ZEIST et W. A. CASPARIE éd., Plants and Ancient Man. Studies in Palaeoethnobotany, Rotterdam, 1984, p. 331-338 ; M.-P. RUAS, « Légumes, épices et fruits consommés au presbytère de Rigny dans la première moitié du XVI $\mathrm{XI}^{\mathrm{e}}$ siècle : données carpologiques », dans E. ZADORA-RIO et H. GALINIÉ et al., « La fouille de l'ancien centre paroissial de Rigny (commune de Rigny-Ussé, Indre-et-Loire). Deuxième rapport préliminaire (1992-1994) », Revue Archéologique du Centre de la France, 34 (1995), p. 229-246.

12. M.-P. RUAS et al., « Archaeobotanical Research... », p. 422. 
dont les semences ont été apportées dans les récoltes et dans les déjections des animaux domestiques à partir des espaces de production (adventices des cultures) et de circulation des troupeaux et des habitants (lieux de pâtures et d'affouragement, chemins). L'espèce prédominante du spectre général est l'arganier, à la fois sous forme de «coques » entières ou de fragments de coque et à l'état de charbons de bois. Arbre emblématique du monde amazigh du sud-ouest marocain, on extrayait de ses graines l'huile d'argan, ressource oléagineuse vitale. Son bois servait pour le bâti, le combustible et les outils, et ses fruits et son feuillage étaient pâturés par les chèvres ${ }^{13}$. Les autres essences ligneuses les plus fréquentes sont le thuya de Berbérie et le laurier-rose utilisés pour la construction et comme combustible. La palette alimentaire, représentée par les restes de graines et de fruits (semences), est dominée par trois céréales, l'orge vêtue, le blé nu et le sorgho, et plusieurs fruits, en particulier le raisin, les dattes et les figues. La féverole et la gesse chiche sont les seules légumineuses ; cette dernière pouvant aussi avoir été une mauvaise herbe des cultures ${ }^{14}$ (tableau 1, fig. 3).

\begin{tabular}{|c|c|c|}
\hline & Type de restes & Total nmi \\
\hline PLANTES CULTIVÉES & & \\
\hline Céréales grains (Cerealia, Orge vêtue, blé nu, sorgho) & grain & 1145 \\
\hline Céréales vannes (Cerealia, Orge vêtue, blé nu, sorgho & éléments d'épis & 264 \\
\hline Légumineuses (Gesse chiche, Féverole et autres indéterminées) & graines & 16 \\
\hline Légume vert (Gourde calebasse) & graines & 1 \\
\hline \multicolumn{3}{|l|}{ Fruits } \\
\hline Ceratonia siliqua, Caroubier & graines & 29 \\
\hline Ficus carica, Figuier & pépins, fruits, pdc & $28+4 \mathrm{fr}+8 \mathrm{pdc}$ \\
\hline Phoenix dactylifera, Dattier & graines & 30 \\
\hline Prueus $c f$. mahaleb, $c f$. Cerisies de Sainte-Lucie & noyau & 1 \\
\hline Punica granatum, Grenadier & pépins & 4 \\
\hline Vitis vinifera, Vigne & pépins, baies \& pdc & $109+9 \mathrm{fr}+32 \mathrm{pdc}$ \\
\hline Ziziphus jujuba, Jujubier commun & noyaux & 2 \\
\hline \multicolumn{3}{|l|}{ PLANTES CULTIVÉES OU SAUVAGES CUEILLIES } \\
\hline \multicolumn{3}{|l|}{ Rives des oueds } \\
\hline Vitex agnus-castus ( graines aromatiques ?), Gattilier & graines & 4 \\
\hline \multicolumn{3}{|l|}{ Plante rudérale ou légume vert? } \\
\hline Beta vulgaris, Bette & fruit & 1 \\
\hline \multicolumn{3}{|l|}{ Pâtures/terrasses horticoles irrigées/Champs de céréales } \\
\hline Argania spinosa, Arganier & fg de coques, graines & 1188 \\
\hline Ziziphus lotus, Jujubier lotier & noyaux & 9 \\
\hline PLANTES HERBACÉES SAUVAGES & graines \& fruits & 2016 \\
\hline
\end{tabular}

Tableau 1. Liste et dénombrement des plantes attestées

par les semences carbonisées dans l'ensemble des contextes d'Igīlīz

fg : fragment ; fr : fruit ; pdc : pedicelle. Les nombres sont donnés en nmi (voir le texte).

13. Ibid., p. 429.

14. La lentille et le pois n'ont pas encore été définitivement reconnus en raison de leur ressemblance à l'état de cotylédon carbonisé avec les graines très polymorphes de la vesce (Lens/Vicia et Pisum/Vicia). 


\section{$\mathrm{nmi}=\mathbf{4 9 0 0}$}

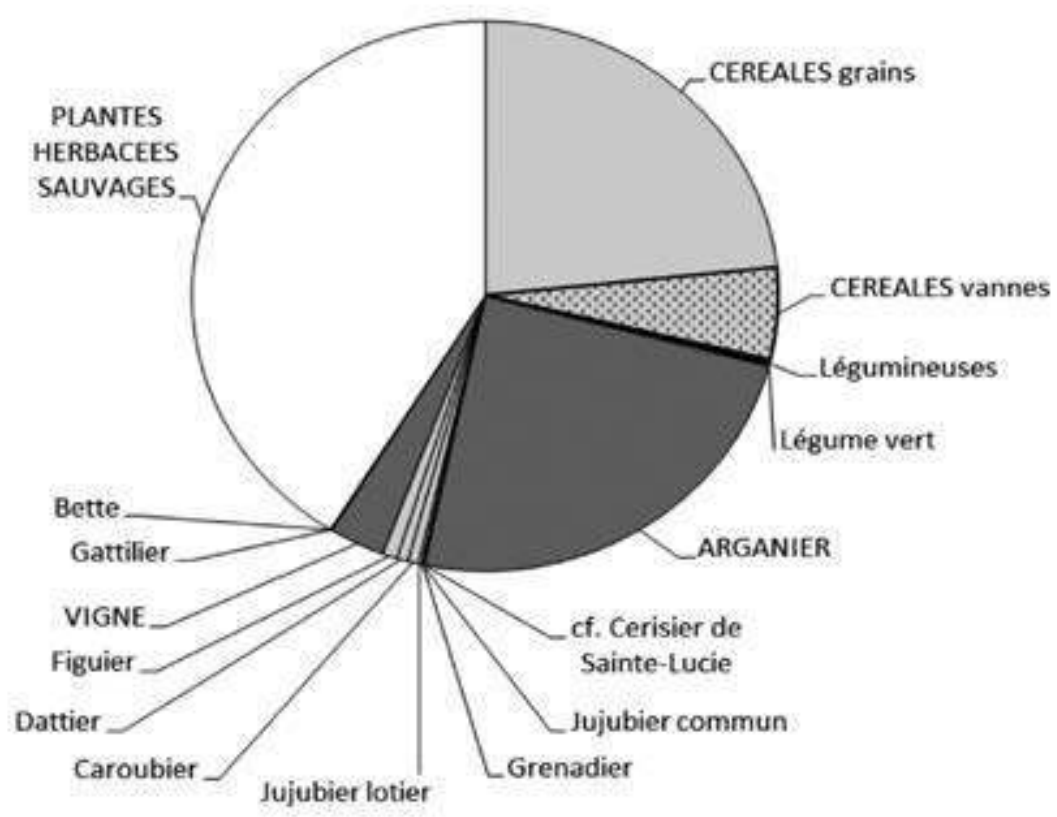

Fig. 3. Spectre général des plantes enregistrées par les semences carbonisées

Dans les trois latrines échantillonnées, seule la fosse de la première cour de la Mḥadra a livré d'abondants restes carpologiques minéralisés et quelques vestiges carbonisés; les deux autres latrines ne contenaient que des restes carbonisés en petites quantités, malgré un volume de sédiment important pour celui de la zone 8 (Latr/VIII-01) ${ }^{15}$. Les latrines de la Mḥadra contenaient aussi des restes d'origine animale non brûlés : vertèbres et pièces buccales de petits poissons d'eau douce ${ }^{16}$, coprolithes indéterminés et pupes minéralisées d'insectes coprophages.

Les trois contenus offrent un spectre général de 18 plantes, dont 9 espèces sont alimentaires (céréales, fruits, oléagineux et condiment/ aromatique) et 9 sont des plantes sauvages des espaces cultivés et parcourus (tableau 2). Le spectre établi à partir des semences minéralisées enregistre des graines de grenade, de câpre et de plantes sauvages que n'ont pas livrées les assemblages carbonisés. Notons que la câpre est attestée sur l'ensemble du site uniquement par trois graines extraites des seules latrines de la Mḥadra

15. L'étude anthracologique des contenus est en cours par S. Ivorra, UMR 5455, CNRS.

16. Détermination B. Clavel, UMR 7209, CNRS, chargé de l'étude de la faune. 


\begin{tabular}{|c|c|c|c|c|}
\hline $\begin{array}{l}\text { RESTES MINERALISÉS } \\
\text { SEMENCES }\end{array}$ & $\begin{array}{c}\text { structure } \\
\text { échantillon } \\
\text { datation } \\
\text { volume traité (I) } \\
\text { type de restes } \\
\end{array}$ & $\begin{array}{c}\text { Latr/V-21 } \\
\text { US } 52113 \\
\text { mil. XII' siècle } \\
5\end{array}$ & $\begin{array}{c}\text { Latr/V-51 } \\
\text { US } 55110 \\
3,5\end{array}$ & \begin{tabular}{|c|} 
Latr/VIII-01 \\
US 80105 \& 07 \\
46,5
\end{tabular} \\
\hline $\begin{array}{l}\text { CËREALES } \\
\text { Cerealia type } \\
\text { FRUITS } \\
\text { Ficus carica } \\
" \text { " } \\
\text { Punica granatum } \\
\text { Vitis vinifera } \\
\quad \text { CONDIMENT } \\
\text { Capparis cf. spinosa } \\
\text { PLANTES SAUVAGES } \\
\text { Bugloissoides arvensis } \\
\text { Chenopodiaceae/Portulacaceae } \\
\text { Euphorbia peplus } \\
\text { Glaucium sp. } \\
\text { cf. Helianthemum } \\
\text { indéterminée }\end{array}$ & 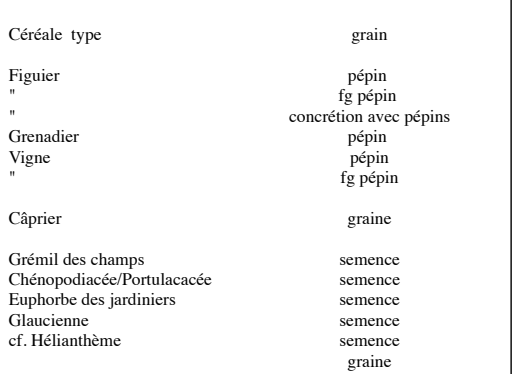 & $\begin{array}{c}6 \\
16680 \\
7750 \\
x \\
33 \\
499 \\
345 \\
\\
3 \\
\\
1 \\
11 \\
1 \\
1 \\
1\end{array}$ & & \\
\hline & $\begin{array}{r}\text { total restes minéralisés carpologiques } \\
\text { total restes minéralisés carpologiques déterminés }\end{array}$ & $\begin{array}{l}25341 \\
25331 \\
\end{array}$ & $\begin{array}{l}\mathbf{0} \\
\mathbf{0}\end{array}$ & $\begin{array}{l}\mathbf{0} \\
\mathbf{0}\end{array}$ \\
\hline $\begin{array}{l}\text { RESTES CARBONISES } \\
\text { SEMENCES }\end{array}$ & $\begin{array}{c}\text { structure } \\
\text { type de restes }\end{array}$ & Latr/V-21 & Latr/V-51 & Latr/VIII-01 \\
\hline 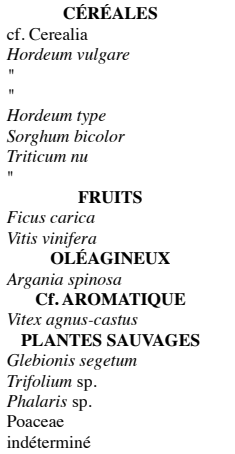 & $\begin{array}{l}\text { cf. Céréale } \\
\text { Orge vêtue } \\
" \\
\text { Orge vêtue type } \\
\text { Sorgho } \\
\text { Blé nu } \\
" \\
\text { Figuier } \\
\text { Vigne } \\
\text { Arganier } \\
\text { Gattilier } \\
\text { Chrysanthème des moissons } \\
\text { Trèfle indéterminé } \\
\text { Phalaris indéterminé } \\
\text { Graminées }\end{array}$ & $\begin{array}{l}4 \\
1 \\
1 \\
1 \\
1 \\
2 \\
1\end{array}$ & 5 & $\begin{array}{l}\mathrm{x} \\
\mathrm{x} \\
\mathrm{x} \\
\mathrm{x}\end{array}$ \\
\hline & $\begin{array}{r}\text { total nombre de semences carbonisées } \\
\text { total nombre de semences carbonisées déterminées }\end{array}$ & $\begin{array}{l}21 \\
20\end{array}$ & $\begin{array}{l}6 \\
6 \\
\end{array}$ & $\begin{array}{l}\text { nd } \\
\text { nd }\end{array}$ \\
\hline $\begin{array}{l}\quad \text { CHARBONS DE BOIS } \\
\text { Argania spinosa } \\
\text { Fabaceae } \\
\text { Nerium oleander } \\
\text { Tetraclinis articulata } \\
\text { Vitis vinifera } \\
\text { indéterminable }\end{array}$ & $\begin{array}{l}\text { Arganier } \\
\text { Légumineuse ligneuse } \\
\text { Laurier-rose } \\
\text { Thuya de Berbérie } \\
\text { Vigne }\end{array}$ & $\begin{array}{c}123 \\
14 \\
1 \\
6 \\
3 \\
3\end{array}$ & & \\
\hline & $\begin{array}{r}\text { total nombre de charbons de bois } \\
\text { total nombre de charbons de bois identifiés }\end{array}$ & $\begin{array}{l}150 \\
177\end{array}$ & $\begin{array}{l}\mathbf{0} \\
\mathbf{0}\end{array}$ & $\begin{array}{l}\mathbf{0} \\
\mathbf{0}\end{array}$ \\
\hline
\end{tabular}

Tableau 2. Identification et dénombrement des restes extraits dans les latrines d'Igīlìz

nd : restes non dénombrés. 
(fig. 4a-e). Les pépins de figue et de raisin apparaissent principalement à l'état minéralisé dans Latr/V-21, alors que des pépins carbonisés des deux fruits composaient une partie du contenu de Latr/VIII-01. Les milliers de pépins minéralisés de figue et de raisin dans ces latrines sont associés à des concrétions de matière organique amorphe dans lesquelles sont inclus des pépins de figue et à des pupes d'insecte coprophage (fig. 4f-i). De tels assemblages caractérisent un milieu organique qui a été investi par la micro-faune détritivore et dont la matrice organique s'est asséchée. La nature fécale des matières accumulées est suggérée par le type de semences réunies, principalement des pépins et petites graines, mais aussi les infimes ossements de petit poisson qu'un organisme humain peut ingérer. Toutefois, la plupart des latrines, notamment en milieu urbain, contiennent une variété de déchets domestiques, depuis les cendres des foyers et fours jusqu'à la vaisselle de table ou de cuisine. Des semences de gros calibre qui proviennent des rejets d'origine culinaire ou des reliefs de repas y sont couramment déversées : noyaux et coques de fruits. Ils sont absents dans les fosses d'Igīlīz.

Si l'on s'en tient à la quantité prédominante de pépins, on pourrait supposer que la figue et le raisin jouaient un rôle de premier plan dans l'alimentation des utilisateurs des latrines de la Mhadra. Les occurrences de ces deux fruits pour l'ensemble des secteurs ne démentent pas cette déduction. Loin après l'argan et les céréales, le raisin, la figue et la datte sont les fruits les plus fréquents parmi les déchets carbonisés. Mais en considérant le nombre de pépins produits par fruit (en moyenne $1500 \mathrm{ou}$ plus), le total conservé dans le comblement de la fosse ne correspond qu'à un rejet équivalant à 14 figues ${ }^{17}$. Un calcul similaire peut être effectué pour les pépins de raisin. Une baie renfermant environ 2 à 3 pépins, parfois 4 , le total des pépins minéralisés renvoie à 200 ou 300 baies, soit quelques grappes. La grenade est aussi attestée par une trentaine de pépins minéralisés ; soit un ou deux fruits (fig. $4 \mathrm{j}$-k). Des grains de probables céréales complètent ce spectre alimentaire.

Cette relative pauvreté en témoins alimentaires et la petite gamme de végétaux enregistrés suggèrent que le comblement conservé est l'ultime résidu de curages auxquels s'est ajoutée la dégradation des matières pendant leur minéralisation. Ces opérations qui permettaient de vider régulièrement la fosse relativement peu profonde sont fortement suggérées par l'absence de système d'évacuation des eaux usées.

17. Un premier calcul a été réalisé pour rapporter le nombre de fragments de pépins à un nombre minimal d'individus entiers (nmi). On obtient un nombre minimal de pépins conservés égal à 20555 (soit 16680 pépins entiers extraits +7750 fragments/2). Le total de ce nmi a ensuite été divisé par 1500 , nombre de pépins par figue. 
Fig. 4. Restes minéralisés des latrines Latr/V-21 US 13, quartier de la Mḥadra et référentiels
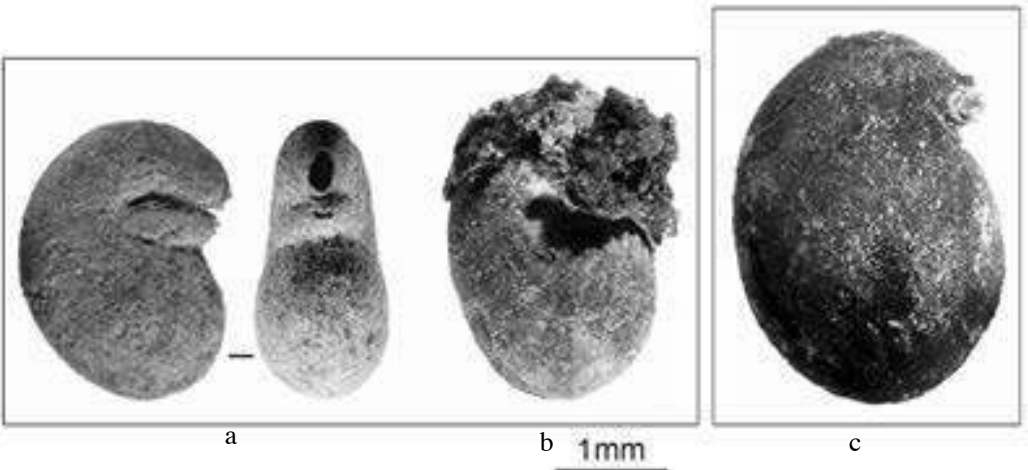

a, b. Deux graines minéralisées de câpre (Capparis cf. spinosa).

c. Une graine de câpre actuelle (Capparis spinosa).

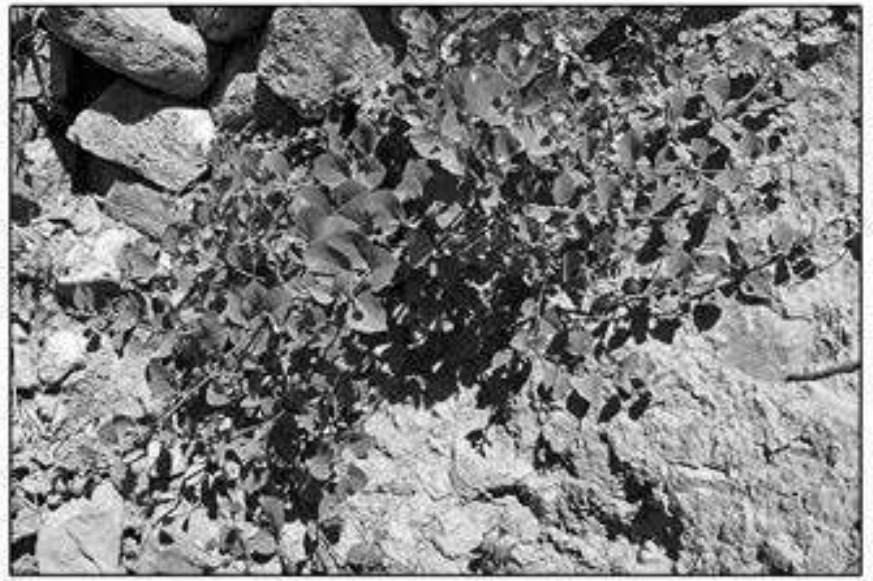

d. Câprier dans un jardin de Tifigit, avril 2014.

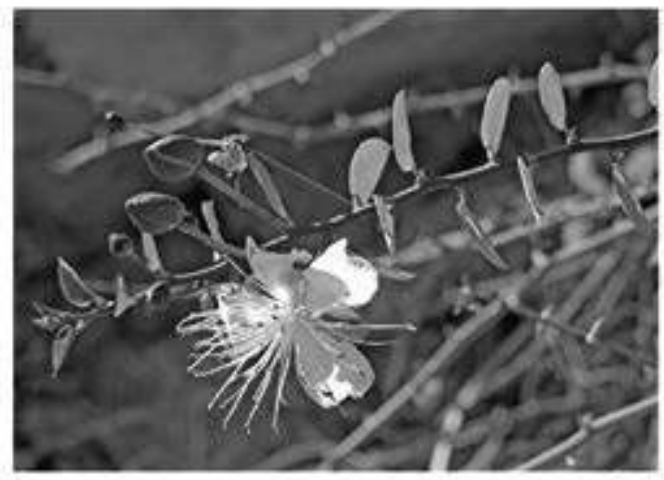

e. Boutons floraux et fleur de câprier, Tifigit, avril 2016. 

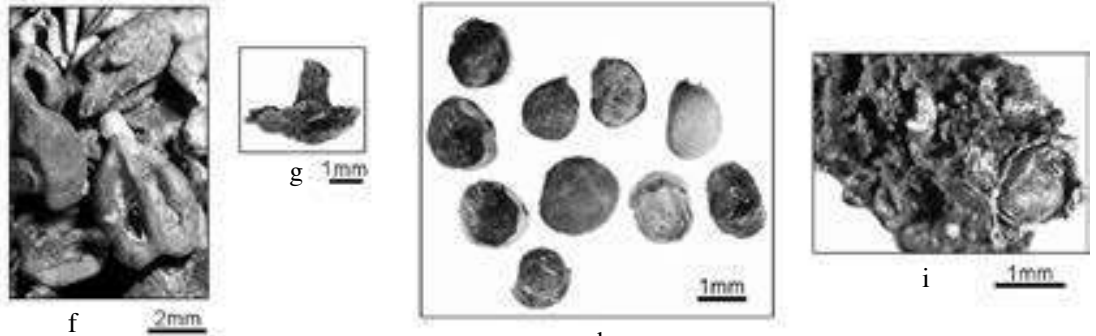

f. Pépins minéralisés de raisin (Vitis vinifera).

g. Pédicelle minéralisé de baie de raisin.

h. Pépins minéralisés de figue (Ficus carica).

i. Concrétion de matière organique avec inclusion d'un pépin de figue.
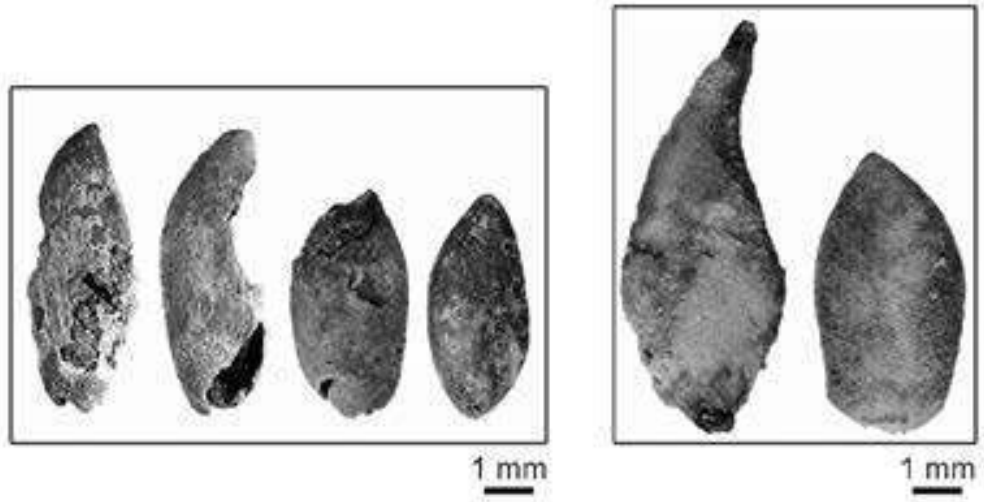

j. Pépins minéralisés de grenade (à gauche) et pépins actuels de grenade (Punica granatum) (à droite).

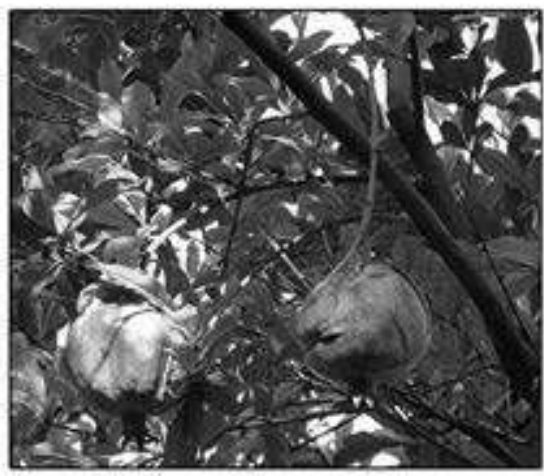

k. Grenades dans un jardin de Tifigit, avril 2014. 
À Igīlīz, des cendres ont aussi été vidangées dans les trois latrines. Celles de la Mḥadra se composaient de grains et parties d'épi des trois céréales de la base alimentaire (orge vêtue, sorgho et blé nu) à côté de pépins de figue et d'un fragment de coque d'argan. Des semences de plantes sauvages typiques des aires cultivées avaient dû y être associées dans les déchets de nettoyage des récoltes (tabl. 2). Les quelques graines de plantes sauvages carbonisées et minéralisées dans la fosse de la Mhadra trahissent aussi le rejet d'autres végétaux issus d'activités qui demeurent imprécises. Ainsi, l'enregistrement végétal des latrines complète celui des foyers dans lesquels dominent les céréales et les légumineuses.

\section{Palette alimentaire végétale et statut social}

\section{Approvisionnement et formes de consommation?}

En dépit de la disparition probable de la plupart des restes, les ultimes témoins de la palette alimentaire des utilisateurs des latrines de la Mḥadra réunissaient au moins trois fruits sucrés à pulpe, trois céréales, une plante oléagineuse et un fruit condimentaire : raisin, figue, grenade, orge, sorgho, blé nu, argan et câpre. Les deux autres fosses offrent un éventail similaire à partir des restes carbonisés. Mais peut-être faut-il ajouter à cette palette les fruits aromatiques du gattilier (Vitex agnus-castus) à la saveur poivrée, présents dans les latrines de l'habitat extra-muros en zone 8, mais sous forme carbonisée ? En effet, le gattilier est un arbuste qui se développe en bord de cours d'eau. Dans l'environnement d'Igīlìz, on le rencontre en fond de vallée sur les grèves exondées de l'oued Arghen. La présence de ses fruits dans des rejets cendreux suppose que des parties de cet arbuste ont été rapportées depuis la vallée ou bien qu'il était planté dans les jardins potagers ou encore qu'il était planté en pot $^{18}$ près des maisons, profitant ainsi d'arrosages suffisants.

La forme de consommation des quatre végétaux identifiés est difficile à démontrer dans la mesure où aucun indice de transformation n'est repérable. Mais le type de reste rejeté et la comparaison avec les assemblages carbonisés peuvent aider à éclairer des pratiques. Dans quelques dépôts de foyers, les pépins de raisin et de figue sont mêlés à quelques fragments des fruits et leurs pédicelles. Les grenades n'ont livré que des pépins. Ces vestiges correspondent à des rejets de fruits consommés entiers, frais ou secs, et dont on recrache les pépins et les queues (pédicelles) réunis

18. L'hypothèse peut être avancée suite à la découverte de fragments de pots à fond percé, dégagés dans les secteurs d'habitat de la zone 2 et la basse-cour de la Qaṣba. Le fond percé suggère un emploi comme pot pour des plantations. 
ensuite dans les cendres lors des balayages du sol. Toutefois, une étude plus détaillée sur les composants de raisin et leur aspect conservés dans les assemblages d'Igīlīz devra être réalisée pour vérifier si certains vestiges ne pourraient pas correspondre à des résidus de pressurage. En effet, selon la description qu'en donne al-Idrīsī dans son ouvrage daté du milieu du $\mathrm{XII}^{\mathrm{e}}$ siècle, les populations du Sous élaboraient une boisson alcoolisée à partir du moût de raisin :

[...] Ils font usage d'une boisson appelée ānzîz, agréable au goût et plus enivrante encore que le vin, parce qu'elle est plus forte et plus spiritueuse ; pour la préparer, ils prennent du moût de raisin doux et le font bouillir jusqu'à ce qu'il n'en reste que les deux tiers dans le vase ; ils le retirent alors du feu, le mettent en cave et le boivent. Cette boisson est tellement forte qu'on ne saurait en faire usage impunément sans y ajouter la même quantité d'eau. Les habitants du Sous en considèrent l'usage comme permis tant qu'elle ne cause pas une complète ivresse $[\ldots]^{19}$.

L'ascétisme et le rigorisme prônés par le nouvel ordre religieux d'Ibn Tūmart ne permettaient sans doute pas l'usage d'une telle boisson sur la montagne. Mais il est permis de supposer d'autres formes de consommation des fruits à pulpe que l'ingestion directe sans préparation.

De la présence de graines de câpres, on déduit que c'est le fruit qui avait été ingéré plutôt que le bouton floral que nous consommons aujourd'hui confit dans le sel ou le vinaigre. Or d'après le traité d'Ibn al- 'Awwâm, auteur arabo-andalou du XII ${ }^{\mathrm{e}}$ siècle, s'appuyant sur une mention dans l'Agriculture nabatéenne, plusieurs façons de les consommer ont pu favoriser le rejet de graines dans la fosse. En effet, on mangeait les fruits verts séchés après les avoir confits dans du vinaigre salé, puis lavés. Ces câpres confites étaient dégustées trempées dans du miel ou du sirop de dattes, ou bien cuites avec la viande. Des câpres non confites étaient aussi préparées avec des substances sucrées $^{20}$. Ces recettes suggèrent que les câpres ne sont pas toujours le condiment des plats salés, mais qu'elles sont aussi des fruits comme les autres dans l'alimentation. L'auteur andalou renseigne aussi sur l'origine des câpres récoltées. Spontanés dans les milieux secs, les câpriers étaient arrachés et plantés dans les jardins. L'irrigation et la fumure amélioraient significativement la saveur des fruits qui perdaient un peu de leur amertume et produisaient moins de graines.

19. AL-IDRĪ̄Ī (mort en 1165), Kitāb Nuzhat al-muštāq fì ihtirāq al-āfāq : AL-IDRĪSī, Livre du divertissement de celui qui désire parcourir le monde, éd. et trad. H. SADOK, Paris, 1983.

20. IBN AL-'AwwĀm, Kitāb Al-Filāha : IBN AL-'AwwÂM, Le Livre de l'agriculture, éd. M. El FAÏZ, trad. J.-J. ClÉMENT-MulLet, Arles, 2000, p. 750. 
Si un approvisionnement lointain de certaines denrées végétales n'est pas à exclure ${ }^{21}$, il est intéressant de constater que des pratiques de transplantation de végétaux sauvages, comme le câprier, peut-être aussi le gattilier, permettaient d'augmenter la diversité vivrière produite près des lieux de vie.

\section{Des régimes alimentaires à lgïlīz?}

Les points de comparaison archéobotaniques avec d'autres sites ruraux n'existent pas pour le Maroc médiéval. Les études actuellement disponibles sont partiellement publiées ou inédites et proviennent en majorité de sites urbains du nord-ouest du Maroc. Il s'agit de trois sites où l'occupation islamique succède à de longues occupations antiques et d'une ville créée à la période médiévale : réoccupation ponctuelle à Lixus $^{22}$, quartier des bains et du palais d'époque idrisside (VIII $-I^{\mathrm{e}} \mathrm{X}^{\mathrm{e}}$ siècle) à Volubiliss ${ }^{23}$, réoccupation du tell à Rirha (IX ${ }^{\mathrm{e}}$-XIV ${ }^{\mathrm{e}}$ siècle $)^{24}$ et le site urbain d'al-Bașra ${ }^{25}$. Dans le sud-est, seule la cité de Sijilmasa, créée au VIII ${ }^{\text {e }}$ siècle et abandonnée au XIV ${ }^{\mathrm{e}}$ siècle, a bénéficié d'une étude des restes de plantes jamais publiée ${ }^{26}$.

Les niveaux islamiques de ces sites placent l'orge vêtue, les blés nus et la féverole comme les grains farineux de la base alimentaire des populations citadines du Maroc, voire de l'ensemble du bassin méditerranéen depuis au moins l'Antiquité ${ }^{27}$. Notons que les résultats carpologiques obtenus sur des sites urbains islamiques du nord-est de la péninsule Ibérique donnent

21. L'identification d'un reste de poisson marin dans l'un des dépotoirs du secteur voisin de la Mhadra montre l'apport extra-régional de denrées animales (travaux en cours B. Clavel, UMR 7209, CNRS, et H. Monchot, Labex Resmed, UMR 8167).

22. G. PÉREZ JORDÀ, " Estudio paleocarpológico », dans C. ARANEGUI GASCó et M. HABIBI éd., Lixus-2. Ladera sur. Excavaciones arqueológicas marroco-españolas en la colonia fenicia. Campañas 2000-2003, Saguntum, extra 6, (2005) p. 221-227.

23. D. Fuller, «Archaeobotanical Work, 2004 », dans E. Fentress, H. LimANE et G. PALumbo éd., Excavation and Conservation at Volubilis, 2004, rapport en ligne : www. sitedevolubilis.org/www/english/about/reports/Report2004.pdf.

24. M.-P. RuAs, I. Figueiral, C. Heinz et S. Ivorra, « Paléoenvironnement et paléoéconomie d'après l'anthracologie et la carpologie », dans L. CALLEGARIN, M. KBIRI Alaoul et A. IchKHAKH éd., Le Site antique et médiéval de Rirha (Sidi Slimane, Maroc). Campagnes de fouilles 2005-2009, Casa de Velázquez-INSAP (Rabat), monographie sous presse.

25. N. MAHONEY, «Agriculture, Industry and the Environment : Archaeobotanical Evidence from Al-Bașra », dans N. BENCO éd., Anatomy of a medieval Islamic Town : Al-Bașra, Morocco, British Archaeological Reports, Serie S1234 Archaeopress, Oxford, 2004, p. 31-42.

26. N. MAHONEY rapports inédits, 1993, 1994 et 1995, aimablement transmis par C. Capel et consultables avec l'autorisation du responsable des fouilles, Ronald A. Messier.

27. M.-P. RUAS et al., «Paléoenvironnement et paléoéconomie... », sous presse. 
la lentille comme seule légumineuse attestée ${ }^{28}$. Les fruitiers enregistrés par leurs graines ou leurs fruits comptent cinq espèces cultivées et trois dont les fruits sauvages étaient récoltés : figuier, jujubier commun, olivier, palmierdattier, vigne, chêne, jujubier lotier et palmier nain. Cette gamme n'est pas toujours aussi diversifiée selon les sites. Les pépins de figue et de raisin sont récurrents et accompagnés d'olives à Volubilis ${ }^{29}$ et d'olives et de dattes à Sijilmasa $^{30}$. À Rirha, les restes de six fruits sont enregistrés dans les niveaux islamiques ${ }^{31}$.

À Igîlīz, l'absence de graines de dattes dans les latrines est probablement due à leur utilisation systématique comme combustible comme le révèlent les fours et les foyers qui contiennent souvent leurs débris. Les latrines délivrent un spectre fruitier aussi étroit que les ensembles des deux villes islamiques d'al-Basra et Sijilmasa, pourtant en prise directe avec les commerces transsaharien et transméditerranéen. Curieusement ni la grenade ni la caroube ni le jujube n'apparaissent dans les déchets alimentaires de ces deux cités, alors que ces fruits étaient consommés sur la montagne d'Igīlīz. Plutôt que le commerce, les aménagements des versants pour les productions locales ont sans doute favorisé l'accès à ces fruits. Mais peut-être n'étaientils pas destinés à tous les résidents de la forteresse. Comment interpréter, en effet, la présenceexclusive de leurs restes et d'un légume, comme la gourde, dans certains secteurs de l'acropole ? Les pépins de grenade et l'unique graine de gourde ne sont attestés que dans la Mhadra (latrines, et four de la cour 21) et deux structures culinaires d'habitats mitoyens. Des noyaux de jujube cultivé ne sont notés que dans un foyer d'une pièce de service de la Qașba et des graines de caroube dans le même foyer, les mêmes cendres que les habitats proches de la Mhadra et deux foyers de celle-ci. Malgré un échantillonnage important, la rareté de leurs occurrences serait-elle l'indice d'un statut particulier de ces denrées qui auraient été destinées à un groupe social privilégié non paysan, à savoir les notables du pouvoir religieux et leur entourage?

La ressource oléagineuse vitale à Igīlīz était l'huile d'argan : aucun noyau d'olive ni graine d'amande ou de noix ne sont attestés. Cette huile, obtenue après de longues heures d'extraction à la meule, était alors réputée, d'après les textes médiévaux du XI ${ }^{\mathrm{e}}$ au XVI ${ }^{\mathrm{e}}$ siècle, comme une production spécifique du Sous et de ses montagnes. Bien que de consommation

28. N. Alonso, F. Antolín et H. KirChNER, « Novelties and Legacies in Crops of the Islamic Period in the Northeast Iberian Peninsula : The Archaeobotanical Evidence in Madina Balagî, Madîna Lârida, and Madîna Turţûša », Quaternary International, 354 (2014), p. 149161.

29. D. FULLER, «Archaeobotanical Work, $2004 »$, p. 9.

30. N. MAHONEY, rapports inédits, 1993, 1994 et 1995, aimablement transmis par C. Capel et consultables avec l'autorisation du responsable des fouilles, Ronald A. Messier.

31. M.-P. RUAS et al., « Paléoenvironnement et paléoéconomie... », sous presse. 
courante pour les populations qui la produisaient, elle était offerte comme cadeau de prestige ${ }^{32}$.

Les catégories des légumes verts et des condiments et aromatiques sont réduites quel que soit le site concerné, en raison de la forme de consommation (feuille) et de l'emploi souvent à l'état broyé de leurs graines moins résistantes à la carbonisation et non déterminables une fois minéralisées.

La faible part de restes alimentaires délivrée par les latrines d'Igīlīz n'autorise aucun parallèle avec les ingrédients d'un régime de frugalité d'ascète ou ceux des règles de la diététique savante du moment. La figue et le raisin sont alors les fruits les plus réputés par les diététiciens d'alAndalus pour leur propriété à améliorer le sang. Les ascètes soufis les consomment principalement séchés ainsi que l'orge ou le sorgho, céréales du monde rural ${ }^{33}$. Ces dernières étaient aussi probablement l'ordinaire des groupes sociaux religieux, militaires et paysans de la montagne d'Igīlīz, comme le montrent les occurrences de leurs grains dans l'ensemble des secteurs du site ${ }^{34}$.

La diversité alimentaire dont profitaient les habitants d'Iḡ̄līz pendant le temps fort de l'occupation de l'acropole durant le XII ${ }^{\mathrm{e}}$ siècle n'avait rien à envier aux populations citadines des plaines fertiles du nord. Pourtant, plusieurs plantes manquent à l'appel par comparaison avec les productions de la vallée du Sous énumérées par al-Idrīsī au XII ${ }^{\mathrm{e}}$ siècle, comme les noix, les coings, les citrons, les pêches, les pommes, le riz et la canne à sucre. Mais il cite aussi les grenades, les figues et le raisin dont les variétés cultivées sont recherchées pour leur qualité.

Malgré les conditions écologiques contraignantes d'une montagne semi-aride, des aménagements de terrasses irriguées ont probablement permis la culture de l'ensemble des denrées consommées, complétées par les plantes sauvages transplantées dans ces lieux artificialisés ou cueillies

32. Les références à l'utilisation de l'huile d'argan d'après les sources arabes médiévales sont réunies dans M.-P. RUASet al., «Archaeobotanical Research... », p. 429-431.

33. J. Salas-Salvadó, M. D. Huetos-Solano, P. Garcíà-Lorda et M. Bulló, « Diet and Dietetics in Al-Andalus », British Journal of Nutrition, 96 (2006), Suppl. 1, p. 100-104 ; H. FERHAT, «Frugalité soufie et banquets de zaouyas : l'éclairage des sources hagiographiques », Médiévales, 33 (1997), p. 69-79.

34. Dans un article consacré au don de nourriture, M. Ghouirghate commente une scène de consommation de l'asmās, par Ibn Tūmart et ses disciples, rapportée par al-Baydaq, compagnon du Mahdī. Ce plat, caractéristique du sud du Maghreb extrême, serait, selon les conclusions de l'auteur, une préparation de grains de céréales bouillis dans un récipient du même nom (« chaudière ») : M. GHOUIRGHATE, «Variations sur le don de nourriture comme instrument de gouvernement chez les Almohades », Horizons Maghrébins, 59 (2008), p. 30-37 (p. 31). Voir également à présent, du même auteur, « Asmās. Le plat emblématique des Almohades », Arabica, 62 (2015), p. 1-22. Sa consommation ritualisée rappellerait la cérémonie du ma 'rūf, repas communautaire dont plusieurs traces matérielles à Igīlīz avèrent la pratique depuis l'époque almohade. 
sur les bords de l'oued dans la vallée, comme le suggèrent à la fois les graines de gattilier mais aussi les petits poissons d'eau douce consommés dans le secteur de la Mhadra.

Les études jusqu'ici menées sur l'habitat rural en al-Andalus - nous manquons cruellement de points de comparaison avec d'autres sites ruraux au Maghreb extrême - montrent bien à quel point les pièces servant de latrines sont rares en milieu rural : les fouilles pratiquées à Alcaria Longa ${ }^{35}$, à Bofilla ${ }^{36}$ ou au Castillejo de Los Guájares ${ }^{37}$ le montrent clairement. De fait, les latrines sont considérées généralement dans l'historiographie comme relevant de « caractères urbains plus que ruraux ${ }^{38}$ », dans le cadre d'un processus de spécialisation des espaces domestiques inhérent à la ville $^{39}$. Certes, on rencontre de tels aménagements sur des sites d'époque almohade considérés comme « ruraux » par les auteurs, comme Yecla ${ }^{40}$ et Villa Vieja de Calasparra ${ }^{41}$, mais les latrines ne semblent apparaître alors que de manière sporadique. Ce phénomène est souvent mis en relation avec la lente pénétration, à partir des XII ${ }^{\mathrm{e}}$ et XIII ${ }^{\mathrm{e}}$ siècles, des standards du confort urbain dans certaines agglomérations rurales (peut-être sous l'influence de l'afflux de réfugiés venus de centres urbains conquis par les Chrétiens plus au nord ?). C'est donc l'adoption du mode de vie urbain, selon A. Bazzana, qui susciterait l'apparition des latrines ${ }^{42}$. Beaucoup plus proche d'Igīlīz sur le plan géographique, l'habitat médiéval étudié dans le Sous-Tekna n'a livré pour l'instant de latrines (et encore : un spécimen sur quatre maisons fouillées) que sur le site de Taghjījt, dans un contexte matériel

35. J. L. Boone, « The First Two Seasons of Excavations at Alcaria Longa : A CaliphalTaifal Period Settlement in the Lower Alentejo of Portugal », Arqueologia Medieval, 1 (1992), p. 51-64, et ID., « The Third Season of Excavations at Alcaria Longa », Arqueologia Medieval, 2 (1993), p. 111-125.

36. J. LÓPEZ ElUM, La alquería islámica en Valencia. Estudio arqueológico de Bofilla, siglos XI a XIV, Valence, 1994, p. 170.

37. M. Bertrand, P. Cressier, A. Malpica Cuello, G. Rossello-Bordoy, « La vivienda rural medieval de 'El Castillejo' (Los Guájares, Granada) », dans La Casa hispano musulmana. Aportaciones de la Arqueología/La Maison hispano-musulmane. Apport de l'Archéologie, Grenade, 1990, p. 207-227 (p. 213-214).

38. A. BAZZANA, Maisons d'al-Andalus. Habitat médiéval et structures du peuplement dans l'Espagne orientale (2 vol.), Madrid, 1992, t. 1, p. 120.

39. Ibid., t. 1, p. 178.

40. L. RuIZ Molina, « Hisn Yakka. Un castillo rural de Šarq al-Andalus. Siglos XI al XIII », numéro monographique de la revue Yakka, 10 (2000), p. 71 et 74.

41. I. Pozo MARTíneZ, « La alquería islámica de Villa Vieja... », p. 175.

42. A. BAZZANA, Maisons d'al-Andalus..., t. 1, p. 193. Le caractère « urbain » des latrines est cependant invalidé par J. NAVARRo PALAZÓN et P. JiMÉNEZ CASTILlo, dans J. NAVARRo PALAZÓn et P. JimÉnez CASTILlo, Siyâsa. Estudio arqueologico del despoblado andalusi(ss. XI-XIII), Grenade, 2007, p. 230-231. 
clairement marqué par une culture urbaine exogène, procédant sans doute de l'État almohade ${ }^{43}$. Cette relation entre présence de latrines dans l'espace domestique et contexte matériel citadin doit cependant être complétée par une indispensable dimension sociologique. Plutôt que d'être un signe de l'abandon du caractère de ruralité, l'adoption d'un tel équipement doit en effet être mise en relation de manière préférentielle avec le processus de densification du bâti. Celui-ci impose de nouvelles contraintes sociales et, par conséquent, une nouvelle discipline des fonctions corporelles, dans un environnement beaucoup moins favorable à l'accomplissement de ces dernières en extérieur. C'est certainement cette recherche d'une plus grande intimité qui explique l'apparition, en divers points du site d'Igīlīz, de latrines. Peut-être la source de la diversité formelle de celles-ci est-elle à chercher dans les origines elles-mêmes variées des occupants des lieux - la communauté charismatique almohade est en effet composée, si l'on en croit les textes, d'étrangers venus de tout le Maghreb et qui se mêlent aux contribules d'Ibn Tūmart. La diversité des configurations laisse à penser parfois à des solutions par défaut, ou nettement contraintes par le bâti préexistant (cas des latrines de la Mhadra et de la grotte 2). Enfin, on ne négligera pas la dimension religieuse que peut également revêtir l'intégration d'une pièce ou d'un réduit destiné à la satisfaction des besoins naturels, mais également à l'accomplissement de la toilette et des ablutions légales. S'agit-il là d'un trait commun à l'habitat rural des régions du Sud marocain aux époques almoravide et almohade ? Ou serait-ce une caractéristique singulière à mettre en rapport avec le statut particulier du site d'Igīlīz, sanctuaire du credo initial de l'almohadisme ? Il est encore trop tôt pour pouvoir faire pencher la balance en faveur de l'une ou l'autre de ces hypothèses.

Le grand intérêt des latrines d'Igīlīz réside par ailleurs dans les restes archéobotaniques qu'elles ont livrés à l'analyse. La mauvaise conservation des restes issus directement des excréments et les curages des latrines ont réduit l'information sur la composition initiale des rejets. Mais, grâce à la diversité des contextes échantillonnés, une image complémentaire entre les assemblages carbonisés et les restes minéralisés permet d'esquisser les grands traits de l'alimentation d'origine végétale sur la montagne d'Igīlīz. Elle se caractérise par une diversité vivrière générale inattendue dans un site rural de montagne où des plantes de cultures exigeantes côtoient des fruits et des légumes sauvages, mais peut-être mis en culture. Le spectre restreint des latrines ne peut servir à caractériser une alimentation particulière.

43. Y. Bokbot, Y. CÁCeres Gutiérrez, P. Cressier, J. De JuAn Ares, M. C. GonzÁlez Marrero, M. A. Hervás Herrera, J. Onrubia Pintado, « Viviendas medievales al sur del Anti-Atlas (Marruecos). Problemas de estudio y especificidades », dans S. GuTIÉRREZ et I. GRAU éd., De la estructura doméstica al espacio social. Lecturas arqueológicas del uso social del espacio, Alicante, 2014, p. 279-298 (p. 294). 
Néanmoins, la rareté de certaines denrées dont les déchets sont limités aux secteurs des résidents religieux invite à discuter de leur statut. Les prochaines investigations dans les habitats proches des entrées, occupées peut-être par des paysans éleveurs, et dans les maisons extra-muros permettront de vérifier cette hypothèse. Le contenu des latrines témoigne par ailleurs d'un emploi multiple comme réceptacle des immondices produites par la vie quotidienne : fosse d'aisance des excreta humains, mais aussi poubelle qui reçoit des amas de plantes herbacées à l'état « frais » (provenant de jonchée ? de litière ?), des cendres évacuées depuis des foyers domestiques (présence de grains de céréales et de déchets de fruits) et, peut-être, des foyers de chauffe des eaux d'ablutions qui utilisaient des bois et des déchets de concassages des noix d'argan. Ces contenus hétérogènes, organiques, servaient-ils à fumer les parcelles de cultures exigeantes - pratique, avec l'irrigation, qui autorisait une production quasi autarcique et diversifiée en céréales et en plantes potagères et fruitières ?

Jean-Pierre Van Staëvel - Université de Paris-Sorbonne - UMR 8167

Marie-Pierre Ruas - UMR 7209, CNRS

Ahmed S. Ettahiri - Insap, Rabat

Abdallah Fili - Université Chouaib Doukkali, El Jadida

Lieux d'aisance et de toilette en milieu rural dans le Maroc médiéval : l'exemple des latrines du site d'lgïliz et les déchets des plantes consommées

Les fouilles menées sur la montagne d'Igīliz (Maroc), épicentre des débuts de la révolution almohade au début des années 1120, a livré jusqu'à présent six structures identifiées comme des latrines. Cet article présente une première synthèse des données archéologiques et archéobotaniques disponibles pour ces lieux d'aisance.

Alimentation et consommation - archéobotanique - latrines - Maroc médiéval - période almohade

Toilet and Bathroom in Rural Areas of Medieval Morocco : the Example of the Latrines of the Site of Igiliz and the Food Plant Waste

Excavations on Mount Igiliz (Morocco), the epicentre of the beginnings of the Almohad revolution in the early 1120s, has delivered so far six structures identified as latrines. This article presents a first synthesis of archaeological data and archeobotanical available for these structures.

Almohad Period - Archaeobotanical Remains - Diet and Consumption Latrines - Medieval Morocco 
